\title{
Light-activated modulation and coupling in integrated polymer-liquid crystal systems
}

\author{
Matthew Proctor, ${ }^{1, *}$ James Bateman, ${ }^{1}$ Keith Daly, ${ }^{2}$ Mark Herrington, ${ }^{1}$ Oleksander Buchnev, ${ }^{3}$ \\ Nina Podoliak, ${ }^{3}$ Giampaolo D’Alessandro, ${ }^{4}$ and Malgosia Kaczmarek ${ }^{1}$ \\ ${ }^{1}$ School of Physics and Astronomy, University of Southampton, Southampton, England, UK \\ ${ }^{2}$ School of Engineering Sciences, University of Southampton, Southampton, England, UK \\ ${ }^{3}$ Optoelectronics Research Centre, University of Southampton, Southampton, England, UK \\ ${ }^{4}$ School of Mathematics, University of Southampton, Southampton, England, UK \\ *Corresponding author: m.proctor@soton.ac.uk
}

Received July 25, 2014; accepted October 7, 2014;

posted October 31, 2014 (Doc. ID 217822); published November 24, 2014

\begin{abstract}
We explore the transfer of an incident light pattern onto the liquid crystal (LC) bulk in a photorefractive cell through an integrated photoconducting layer that modulates the electric field applied to the device. The electrical properties and the strength of modulation are investigated as a function of the incident light intensity as well as the frequency and amplitude of the applied voltage, for two LCs with very different electrical conductivity. A simplified electrical model of the cell is proposed, demonstrating that the LC conductivity is a key factor determining
\end{abstract} the beam-coupling strength. (C) 2014 Optical Society of America

OCIS codes: (160.3710) Liquid crystals; (160.5140) Photoconductive materials; (090.1970) Diffractive optics; (050.1950) Diffraction gratings.

http://dx.doi.org/10.1364/JOSAB.31.003144

\section{INTRODUCTION}

Light valves and optically addressed spatial light modulators are now well-established technologies. Their typical design $[1,2]$ combines a liquid crystal (LC) layer with a photoconductor, whose main function is to optically address and modify the alignment of the LCs. Silicon is the most commonly used photoconductor, but other routes have been explored, involving windows made from photorefractive crystals, such as BSO or $\mathrm{KNbO}_{3}$ [3] . Recent developments in photosensitive organic materials show that polymers are capable of replacing inorganic crystals for seamless, well-integrated all-organic modulators [4]. They are highly responsive to light and also easily processed at low cost. Polymers can also have a tailormade response through the use of dopants, such as polyvinyl-carbazole doped with fullerenes $\left(\mathrm{PVK}_{\mathrm{C}} \mathrm{C}_{60}\right)$ [5] .

Two standard techniques to determine the modulation of the LC alignment and, hence, of the refractive index are beam-coupling and diffraction efficiency measurements. These rely on the formation of a periodic refractive index grating and are not restricted to LCs: they are typical, for example, of photorefractive materials. They are also at the heart of applications such as optical beam deflection, pattern recognition, and coherent image amplification [6]. Here we use these effects to assess the ability of a photoconducting layer to modulate the LC alignment and form a refractive index grating.

Strong refractive index gratings lead to large coupling coefficients, as observed in inorganic crystals such as $\mathrm{BaTiO}_{3}$ and $\mathrm{Rh}: \mathrm{BaTiO}_{3}$ [7]. However, it is not essential to use bulk photorefractive materials to achieve a strong refractive index modulation. More recently, efficient beam coupling was shown in a wide range of materials and devices, including
LC light valves based on organic [ $[\underline{4}, \underline{8}]$ and inorganic [2] photoconductors, polymer-dispersed [9] and ferroelectric LCs [10], and photorefractive polymers $[\underline{11}, 12]$.

The system we study here is a hybrid photorefractive LC cell. This consists of a standard substrate with polyimide (PI), a layer of polyvinyl carbazole (PVK), sensitized with $\mathrm{C}_{60}$ (PVK: $\mathrm{C}_{60}$ ), and a LC bulk sandwiched between them. PVK: $\mathrm{C}_{60}$ is a very efficient system to transfer a modulated light intensity pattern to a refractive index grating in an LC layer. Previous studies have shown that such a device is capable of screening high electric fields $(\sim 30 \mathrm{~V})$ by building double charge layers at the polymer-LC interface [13]. The surface charge layers can then be selectively discharged by light, with a resolution of the order of micrometers. As a result, the orientation of LCs can be selectively switched by illuminating with light intensities as low as $70 \mu \mathrm{W} / \mathrm{cm}^{2}$.

In a hybrid photorefractive LC cell, a light intensity pattern incident on the photosensitive layer can be transferred, given the right conditions, to the LC bulk. This process takes place when both optical and AC frequency $(0.01 \mathrm{~Hz}$ to $1 \mathrm{kHz}$ in the case of our experiment) electrical fields are applied. The superposition of two coherent incident beams on the photoconductor creates a modulation in the conductivity, which, when used in combination with the applied voltage, produces a modulated AC electric field across the cell. This process aligns the LCs and modulates the refractive index.

Typically, beam-coupling or diffraction efficiency experiments in integrated polymer-LC structures have been carried out with an applied DC voltage. However, recently it has been reported that using a slow AC frequency [14] can also lead to the building up of refractive index gratings while avoiding long-term ionic damage of the cells. Moreover, the frequency 
offers an additional control parameter that can lead to enhanced diffraction efficiency with respect to a DC field [14].

In this work we explore how the optical and electric properties of both a photoconductor, such as $\mathrm{PVK}: \mathrm{C}_{60}$, and a nematic LC can be tuned for optimal light-driven modulation. Key to this study is the determination of the voltage applied to the LC layer, as opposed to the (known) voltage applied to the cell. Obtaining the correct voltage drop across different layers as a function of experimental and material parameters is nontrivial, but it is essential in optimizing the performance of such photosensitive cells. One technique for measuring this voltage drop is known as the voltage transfer function (VTF) [15]. In this method, the electro-optical response of the cell to the frequency and amplitude of an applied voltage is measured optically using a familiar cross-polarization experiment, shown in Fig. 1(a). The relative voltage drop across the LC and polymer layers can be determined directly from the resulting cross-polarized intensity maps.

It is natural to expect that the voltage applied to the LC and, hence, the refractive index modulation, is related to the LC conductivity. We have, therefore, performed beam-coupling experiments using E7 and TL205, two LCs with very different electrical conductivities [16]. We have routinely measured beam-coupling efficiencies of approximately 10\% in E7, the more conductive of the two, while in TL205 the beam-coupling efficiencies were approximately two orders of magnitude smaller. The experiments also reveal that there can be two frequency regions where beam coupling is significant. To understand these observations and to estimate the parameter regions for optimal beam coupling we have developed an approximate model of the electrical behavior of the cell and have used it to estimate the cell modulation transfer function (MTF), i.e., the ratio of the modulation of the voltage

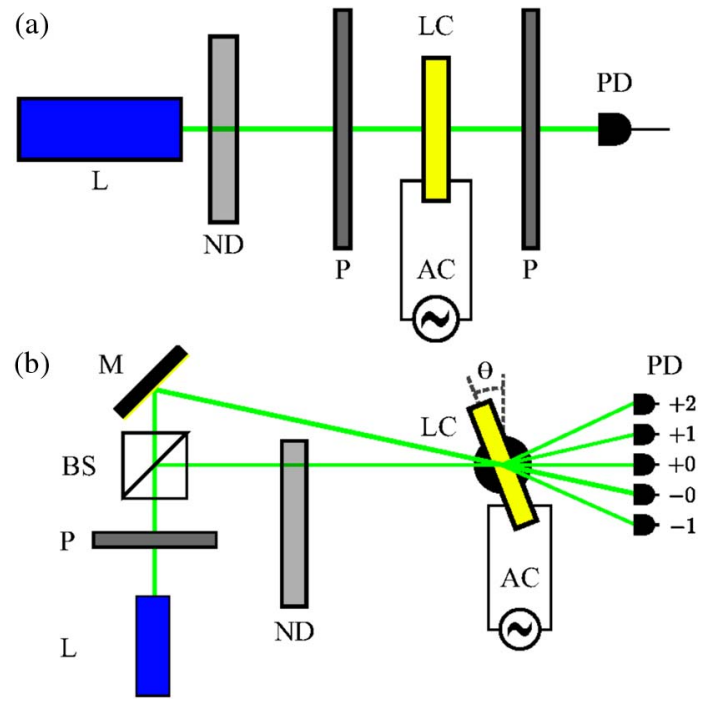

Fig. 1. Schematic diagrams of the experimental setups for measuring (a) cross-polarized intensity and, hence, the voltage transfer function, and (b) intensity of diffracted orders in a two-beam-coupling regime. $\mathrm{L}$, diode-pumped solid-state $532 \mathrm{~nm}$ laser; ND, neutral density filter; $\mathrm{P}$, polarizer; LC, liquid crystal cell (on rotation mount for two-beam coupling); AC, signal generator; PD, amplified photodiode(s), with the diffracted orders identified, the output of which is recorded via an analog-to-digital converter; $\theta$, cell tilt angle; BS, nonpolarizing beam splitter; M, mirror. The polarizers in (a) are rotated by $90^{\circ}$ with respect to each other. applied to the LC and the modulation of the conductivity of the photoconductor. While our investigation is focused on a particular photosensitive polymer and some representative nematic LCs, the characterization approach described here can be applied to generic polymer-LC systems. The approximate MTF we have obtained not only explains the possible presence of two frequency regimes for beam coupling, but also highlights the role that the relative conductivities of the LC and polymer layers have on its strength. The main result of our investigation is that optimal beam coupling requires a careful matching of the resistance of the photoconductive and LC layers.

The structure of this paper is as follows. We begin, in Section 2, by describing the experimental methodology, the main setup, and the most significant measurements. This is followed in Section 3 by an approximate electrical model of the device that is used to interpret the experimental data (Sections 3.B and 3.C). We conclude with a discussion of the limitations on light-driven modulation in photorefractive LC cells and how they may be overcome.

\section{EXPERIMENT}

Our experimental methodology relies mainly on two types of measurements. The first is the determination of the VTF, as a function of applied voltage and frequency, using crosspolarized intensity measurements. The second is the measurement of optical beam coupling and diffraction efficiency into different orders.

\section{A. Experimental Setup}

The hybrid photorefractive LC cells used in our experiments are three-layered planar devices, consisting of a PI alignment layer, a 10-12 $\mu \mathrm{m}$ thick LC layer and a 50-100 nm thick photoconducting $\mathrm{PVK}: \mathrm{C}_{60}$ layer (the details of the cell are discussed in Section 3, and a schematic diagram is shown in Fig. 8).

The planar cells were prepared by spin-coating PI or PVK: $\mathrm{C}_{60}$ onto ITO-coated glass slides. The slides were then heated at $200^{\circ} \mathrm{C}$ for $2 \mathrm{~h}$ and rubbed with a rotating velvet cloth, before being fixed together with UV curable glue mixed with $12 \mu \mathrm{m}$ spacer beads. The completed cells were filled with E7 or TL205 from Merck using capillary forces. This process allowed construction of both non-photo-active PI-PI cells and photorefractive PI-PVK: $\mathrm{C}_{60}$ cells.

The VTF was measured using the same procedure as in Ref. [15], using a $532 \mathrm{~nm}$ laser with a waist of $2.2 \mathrm{~mm}$, which was attenuated by an ND filter wheel. This allowed us to measure the VTF under varying degrees of incident light intensity. When not measuring, the cell electrodes were shorted to equalize any charge carrier buildup in the polymer layers. In addition, the measurement of the intensity was delayed by $3 \mathrm{~s}$ after each voltage step, and by 2 min after the ND filter wheel was rotated in order to minimize experimental drift, mainly due to heating in the ND filter.

The arrangement for the two-beam-coupling experiment was similar, with the following modifications (see bottom panel of Fig. 1): light from the laser was polarized and then split using a nonpolarizing beam-splitter cube. The transmitted light was directed with a mirror to fall coincident with the light reflected by the beam splitter on the LC cell. The inter-beam angle was controlled by changing the distance to the cell, while the ratio of the beam intensities was varied 
by changing the ND filter on one of the two beams. The light polarization was in the plane of the two incident beams, while the LC director at rest was perpendicular to it. The cell was placed on a rotation mount, which allowed rotation around an axis perpendicular to the plane of the beams, and the cell was rotated to $\theta=30^{\circ}$ with respect to the beam bisector, a standard setup that breaks the symmetry between the two beams and allows for significant energy transfer between them. The powers of each of the diffracted orders, including those of the +0 and -0 orders, which correspond to the transmitted beams, were measured using amplified photodiodes connected to a multichannel ADC.

\section{B. Voltage Transfer Function}

A key question in the analysis of photorefractive or photosensitive cells is how much of the voltage applied to the cell is dropped across the LC layer. This is clearly a function of frequency, because each layer is approximately a parallel combination of a resistance with a capacitance, but also of the light intensity, because of the photosensitivity of the PVK: $\mathrm{C}_{60}$ layer. The VTF was measured at different incident intensities to observe the effect that changing these parameters has on the voltage drop.

At high frequency, the cross-polarized intensity at fixed voltage amplitude is essentially stationary, while at low frequency it follows the driving voltage curve. Here we plot the average of the cross-polarized intensity. Some sample plots are shown in Figs. $\underline{2}$ and $\underline{3}$ for standard cells (thickness $12 \mu \mathrm{m} ; \mathrm{PVK}: \mathrm{C}_{60}$ and PI alignment layers on entry and output facets, respectively) filled with E7 and TL205, respectively. We have measured the VTF for different cells, filled with either TL205 or E7, with similar thicknesses and alignment layers. The VTFs of the two LCs have the same qualitative behavior, but differ quite considerably in detail. In particular, the change of the VTF of a TL205 cell with light intensity is

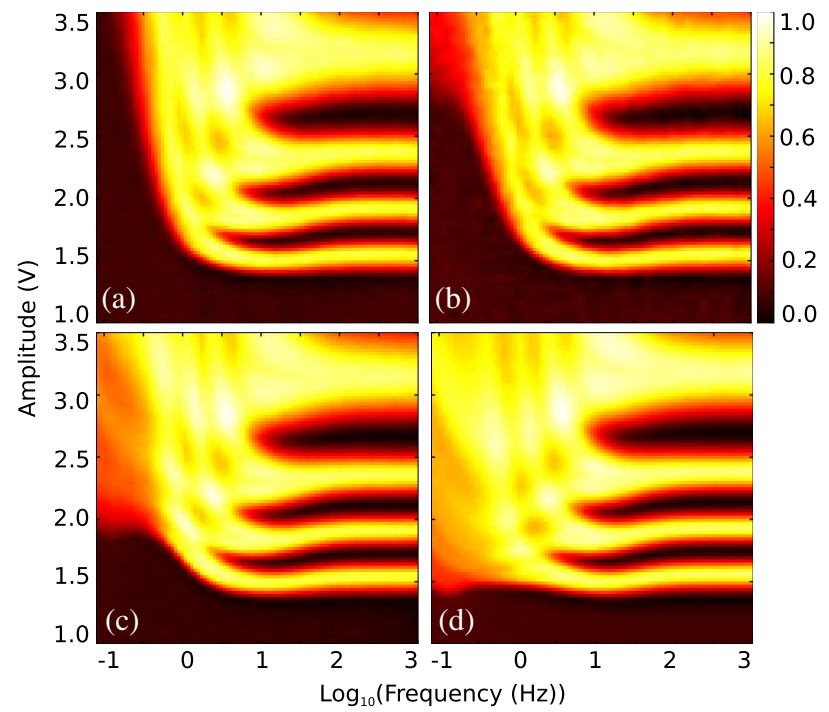

Fig. 2. Voltage transfer function measurements showing the average cross-polarized intensity as a function of the amplitude (vertical) and frequency (horizontal) of the voltage applied to an E7 cell. The four plots show increasing intensity from top left to bottom right: (a) $0.18 \mathrm{~mW} / \mathrm{cm}^{2}$, (b) $1.8 \mathrm{~mW} / \mathrm{cm}^{2}$, (c) $5.7 \mathrm{~mW} / \mathrm{cm}^{2}$, and (d) $68 \mathrm{~mW} / \mathrm{cm}^{2}$. Cell parameters: LC E7, thickness $12 \mu \mathrm{m}, \mathrm{PVK} \mathrm{C}_{60}$ and PI alignment layers on the input and output facets, respectively.

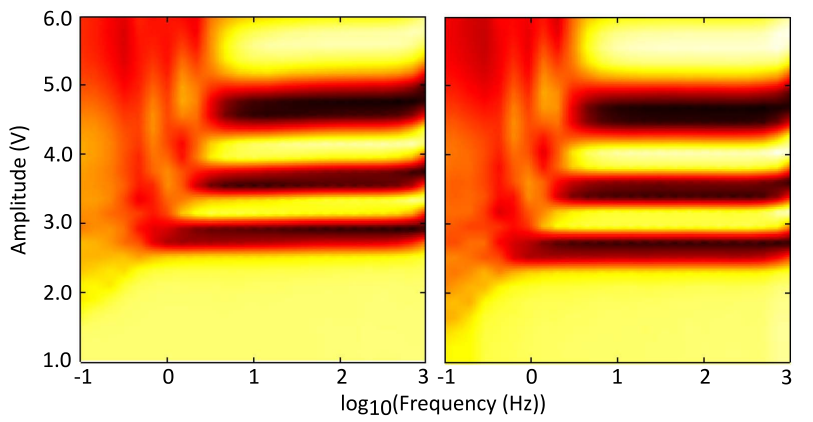

Fig. 3. Voltage transfer function measurements showing the average cross-polarized intensity as a function of the amplitude (vertical) and frequency (horizontal) of the voltage applied to a TL205 cell. Same color coding and color bar as in Fig. 2. The incident intensity was (left) $0.092 \mathrm{~mW} / \mathrm{cm}^{2}$ and (right) $22 \mathrm{~mW} / \mathrm{cm}^{2}$. Cell parameters: LC TL205, thickness $12 \mu \mathrm{m}$, PVK: $\mathrm{C}_{60}$ and PI alignment layers on the input and output facets, respectively.

hardly visible at the resolution of our measurements (see Fig. 3). We attribute this difference to the much higher resistivity of TL205 with respect to E7 [16] so that even in darkness most of the voltage is dropped across the LC layer.

From these plots we can see, for example, that the Frederiks transition, i.e., the voltage at which the cross-polarized intensity starts to change from its $0 \mathrm{~V}$ value, is a function of frequency and light intensity. In Fig. 4 we plot the Frederiks voltage for the E7 cell used in Fig. 2, scaled to its highfrequency, low-intensity value, where we can expect that the voltage applied to the cell is dropped entirely across the LC layer due to its high capacitive impedance. At frequencies as low as $1 \mathrm{~Hz}$ the voltage is dropped almost entirely across the LC layer even at low light intensities. However, as the frequency decreases, up to approximately $60 \%$ of the voltage is dropped across the PVK: $\mathrm{C}_{60}$ layer. Another observation that can be deduced from this plot is that the PI layer plays a secondary role in the electrical behavior of the cell: had this not been true, we would have seen a lightindependent increase in the Frederiks threshold for decreasing frequency.

In the case of TL205 the situation is slightly different (see Fig. 3): as the frequency decreases the Frederiks threshold decreases because the LC responds to the amplitude rather than the rms average of the applied voltage. The decrease is larger in the illuminated cell (right panel of Fig. 3) because the reduced resistivity of the $\mathrm{PVK}: \mathrm{C}_{60}$ layer allows more of the voltage amplitude to drop across the LC.

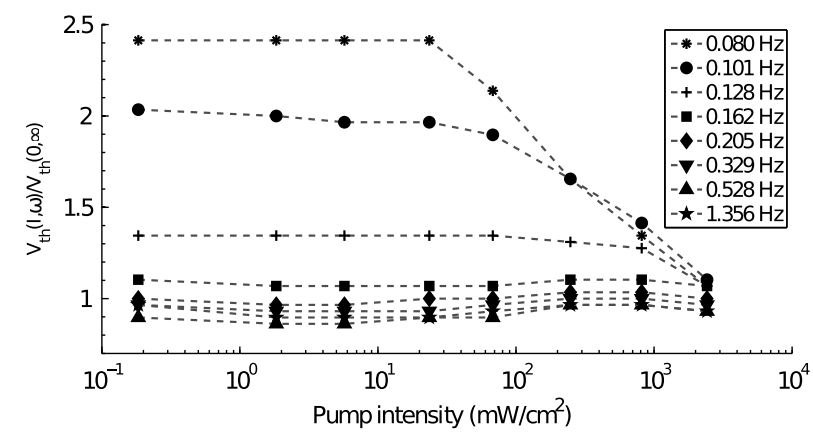

Fig. 4. Frederiks transition as a function of frequency for different light illumination levels for an E7 cell. This plot was obtained from the data used for Fig. 2 . 
In summary, in both cells illumination changes the voltage dropped across the LC layer at low frequency: however, the effect is much smaller in the case of the more resistive LC.

\section{Average Beam Coupling}

We have measured beam coupling for two grating pitch values, $\Lambda \simeq\{2,18\} \mu \mathrm{m}$, corresponding to the Bragg and Raman-Nath regimes, respectively [17]. For the smaller pitch values, we have observed no significant coupling, suggesting that the cell is not able to support such a fine pitch. For the larger pitch, we have observed beam coupling from the fundamental to different orders. We illustrate this in Fig. $\underline{5}$ for a cell filled with TL205.

While the effect of light intensity on a TL205 cell is much smaller than for E7, the effective nonlinearity of the LC cell is such that even a small modulation produces significant, if not large, beam coupling. From Fig. $\underline{5}$ we see that as the voltage is increased above the Frederiks threshold, there is a loss of energy from the fundamental mode that is roughly independent of frequency: we believe that this is due to increased scattering from the cell due to its dynamical response to the applied voltage. As the voltage is increased, there appear to be two different coupling regimes: a weaker low-voltage, low-frequency regime centered at approximately $0.01 \mathrm{~Hz}$ (thin region on the left of the \pm 1 diffracted orders panels in Fig. 5) and a stronger, higher-voltage, higher-frequency regime, centered at approximately $50 \mathrm{~Hz}$. The former asymptotically decreases with increasing voltage, while the latter increases. This behavior is also confirmed by the +2 diffracted order: in this case the low-frequency region is much weaker. This pattern was reproduced in other TL205 cells, but for some of them the low-frequency region was the stronger one. The preparation of the layers and the cells followed the same recipe, but the cells were prepared at different times, over several months. It

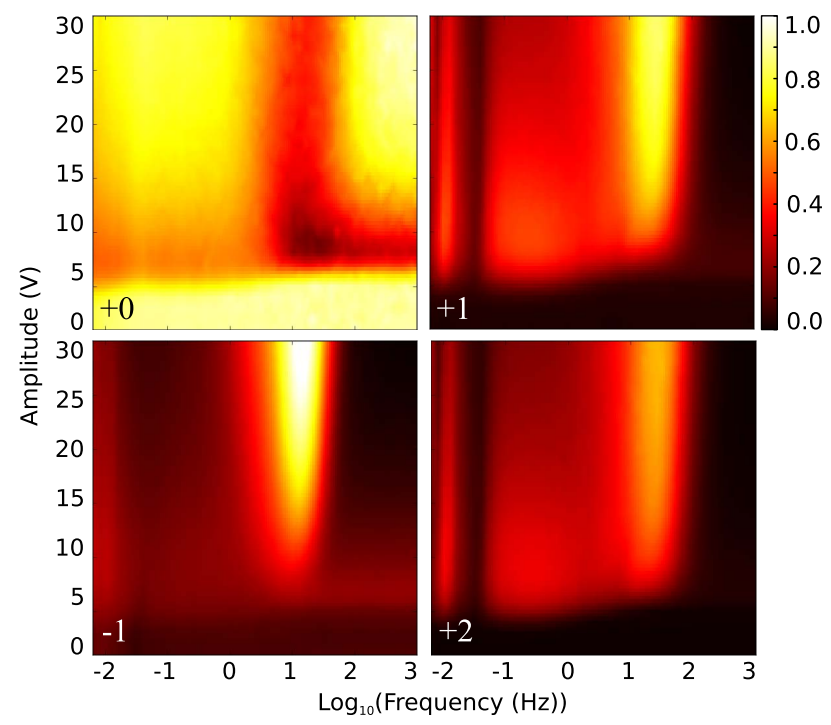

Fig. 5. Intensity of several diffracted orders, including the depleted zeroth order, for a TL205 cell as a function of the frequency and amplitude of the voltage applied to the cell. The color scales cover the following ranges, as fractions of the incident intensity: +0 from 0.9 to $1, \pm 1$ from 0 to $10^{-3}$, and +2 from 0 to $5 \times 10^{-4}$. The beam average intensity was $600 \mathrm{~mW} / \mathrm{cm}^{2}$, and the grating spacing was $18 \mu \mathrm{m}$. Cell parameters: LC TL205, thickness $12 \mu \mathrm{m}, \mathrm{PVK}_{60}$ and PI alignment layers on the input and output facets, respectively. is therefore possible that these differences are due to some changes in or contamination of the raw materials supplied for the experiment. Furthermore, there could be some small variation in the thickness of the PVK: $\mathrm{C}_{60}$ layer or in the $\mathrm{C}_{60}$ doping that may have affected the electrical properties of the photoconducting polymer layer. In all cases, the intensity of the diffracted orders in TL205 is much weaker than the fundamental beam, indicating that while beam coupling is present and the gain per unit length is large (these are very thin devices), the total gain for TL205 remains small.

In the case of $\mathrm{E} 7$, a more conductive LC, the general behavior of the beam-coupling strength with voltage frequency and amplitude is approximately the same as for the TL205 (see Fig. 6). There is only one beam-coupling region, at low frequency (the narrow peak near the Frederiks threshold is likely to be due to enhanced scattering at this voltage). The beamcoupling strength, characterized by the intensity coupled into the \pm 1 order beams, is approximately 50 times larger than in TL205, in agreement with the observation that the VTF function is much more sensitive to light in E7 than in TL205 cells (Fig. 3). This observation indicates that an appropriate matching of the resistances of the photoconducting and LC layers is essential for beam-coupling applications. In Section 3 we use an approximate electrical model of the cell to quantify this statement.

\section{Time-Dependent Beam Coupling}

At low frequencies the LC orientation approximately follows the driving voltage. Measurements of beam coupling as a function of time give insight into the electrical behavior of the cell. In Fig. 7 we show photographs of the beams at the output from the cell in different locations of the cycle for a large voltage amplitude. There are two items to note: the first is that the cell responds differently to the positive and the negative parts of the cycle. This has already been reported in the literature: the mobility of electrons and holes in PVK: $\mathrm{C}_{60}$ is different [18-20], and $\mathrm{C}_{60}$ has different hole/electron acceptor levels [21]. The second observation is that the largest beam coupling is at intermediate voltages. At first impression this is strange, because the analysis of beam-coupling strength in [22] indicates that in the experimental configuration used in these measurements (TM configuration in the notation of [22]) the strength of beam coupling saturates rapidly with voltage, but does not decrease. We will argue in Section 3 that the observed

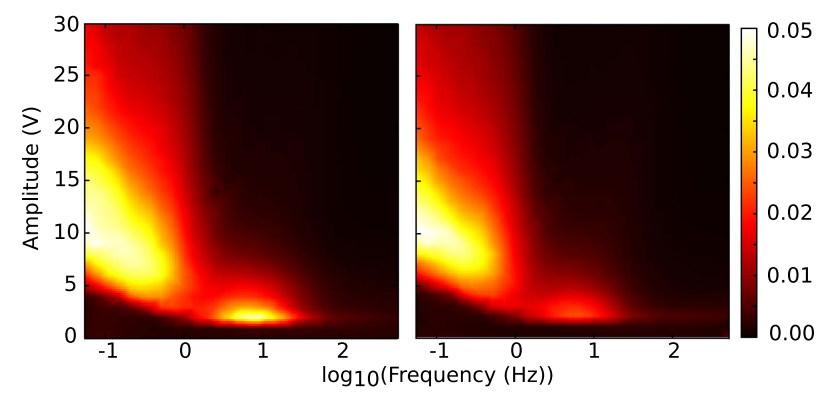

Fig. 6. Intensity of the \pm 1 diffracted orders (left and right, respectively) for an E7 cell as a function of the frequency and amplitude of the voltage applied to the cell. The color scale is normalized to the pump beam intensity with no diffraction. Cell parameters: LC E7, thickness $12 \mu \mathrm{m}, \mathrm{PVK} \mathrm{C}_{60}$ and PI alignment layers on the input and output facets, respectively. The beam average intensity was $600 \mathrm{~mW} / \mathrm{cm}^{2}$, and the grating spacing was $18 \mu \mathrm{m}$. 


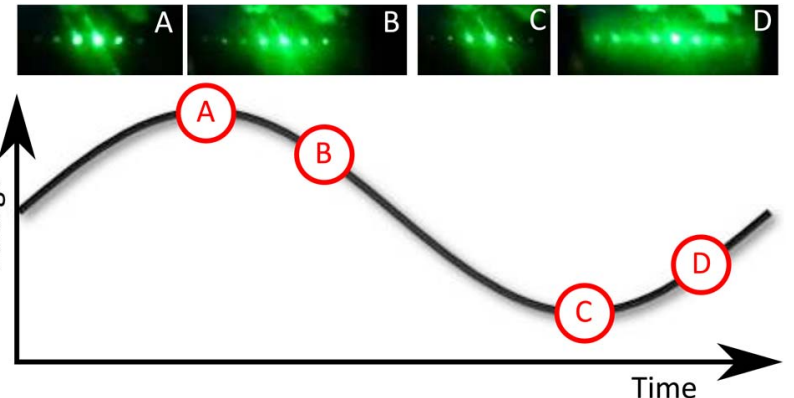

Fig. 7. Snapshots of coupled beams in different parts of the cycle Same cell as in Fig. 2, driving voltage amplitude $28 \mathrm{~V} \mathrm{rms}$ and frequency $0.1 \mathrm{~Hz}$.

reduction in coupling strength is due to a reduction in conductivity of the PVK: $\mathrm{C}_{60}$ layer at high voltage. The voltage dependence of the PVK: $\mathrm{C}_{60}$ conductivity has already been reported in the literature [23] and has been confirmed by plasmon resonance measurements in a system almost identical to ours [24,25].

We conclude this section by noting that the time-dependent measurements in Fig. 7 offer a key to interpret the decrease with voltage amplitude of the low-frequency coupling reported in Fig. 5: as the voltage amplitude is increased, the part of the voltage cycle suitable for beam coupling, i.e., when the voltage is approximately in the range $\pm 10 \mathrm{~V}$, decreases, and hence, the average beam-coupling strength decreases.

\section{MODELING ANALYSIS OF BEAM COUPLING}

In this section we develop an approximate electrical model of a photorefractive LC cell. The aim is not just to interpret and understand the experimental data reported above, but also to assess the role of the LC conductivity for optimal beam coupling. There are already in the literature detailed studies $[22,26,27]$ of the role that the cell parameters, e.g., the cell tilt angle, the LC pretilt, and the anchoring energy, play on beam coupling. There are also detailed measurements of the electrical properties of PVK with or without $\mathrm{C}_{60}$ doping [18-21,28]. These could be coupled with existing models of ion currents in LCs [29-31] and the role of Debye layers [32] at the LCpolymer interface. Here we do not follow this approach for two reasons: first, it is very hard to obtain accurate and reproducible values of the parameters of $\mathrm{PVK}: \mathrm{C}_{60}$ in contact with a LC, so that we would not be able to significantly constrain a model. Second, our aim is to identify generally the limits to beam-coupling strength posed by the conductance of the LC layer with respect to that of a generic photoconducting polymer layer, without restricting our results to the specific device used in the experiments reported in Section 2.

The beam-coupling analysis performed in Ref. [22] indicates that in the regime in which we operate the beamcoupling strength is roughly proportional to the amplitude of the voltage modulation across the LC layer. We therefore model neither the LC alignment nor the ensuing beam coupling, but develop instead a simple model to measure the amplitude of this modulation as a function of the material and cell parameters. Second, we approximate the cell with only three layers, equivalent to PVK: $\mathrm{C}_{60}, \mathrm{LC}$, and $\mathrm{PI}$, each of which is parametrized by a spatially homogeneous dielectric permittivity and an electrical conductivity that is modulated only in the
PVK: $\mathrm{C}_{60}$ layer. This model, while not sufficiently accurate to fit quantitatively experimental results [32], is simple enough to be amenable to a perturbation expansion solution that makes its predictions easier to understand. We outline the model in Section 3.A. We use this to interpret the experimental results in Sections 3.B and 3.C. The details of the model can be found in Appendix $\underline{\mathrm{A}}$.

\section{A. Modulation Transfer Function}

By analogy with the VTF discussed in Section 2.B, we define the MTF, $\Psi$, as

$$
\Psi \equiv \frac{\sigma_{\mathrm{PVK}}}{\Delta \sigma_{\mathrm{PVK}}} \frac{\Delta V_{\mathrm{LC}}}{V},
$$

where $\Delta V_{\mathrm{LC}}$ is the amplitude of the modulation of the voltage at the PVK: $\mathrm{C}_{60}-\mathrm{LC}$ interface, $V$ is the voltage applied to the cell, and $\Delta \sigma_{\mathrm{PVK}} / \sigma_{\mathrm{PVK}}$ is the percentage variation of the PVK: $\mathrm{C}_{60}$ conductivity. The MTF measures the ability of the cell to transfer a modulation of the conductivity of the PVK: $\mathrm{C}_{60}$ layer into a modulation of the voltage at the LC layer. The MTF is an intrinsic property of the cell and can in principle be obtained from the VTF if one were able to measure the percentage change of the conductivity of the $\mathrm{PVK}: \mathrm{C}_{60}$ layer. It can also be estimated theoretically using an electrical model of the cell.

We use a simple model to do this. The details of the model and the full definition and scaling of the parameters are given in Appendix A; here we sketch its main features. We assume that the cell can be represented by three layers (see Fig. 8) with (dimensional) conductivity $\tilde{\sigma}_{k}$ and dielectric permittivity $\tilde{\epsilon}_{k}$, with $k=1,2,3$ corresponding to PVK: $\mathrm{C}_{60}, \mathrm{LC}$, and PI layers, respectively. The conductivity of the PVK: $\mathrm{C}_{60}$ layer is spatially modulated with the period $\Lambda$ of the grating [Eq. (A2)]. Moreover, we assume that the (nondimensional) amplitude $\sigma_{1,1}$ of the modulation is small, so that we can consider it as a perturbation. There are two justifications for this hypothesis: first we are interested in obtaining a formula that will give us some understanding of the beam-coupling strength, rather than a quantitative description. Second, the measured beam-coupling strength in our experiments is relatively small, which suggests that the modulation of the LC and of the voltage across it is also small.

The potential in the cell is a solution of the conservation of current equation (A1) and can be solved for using a perturbation expansion in the strength of the modulation of the

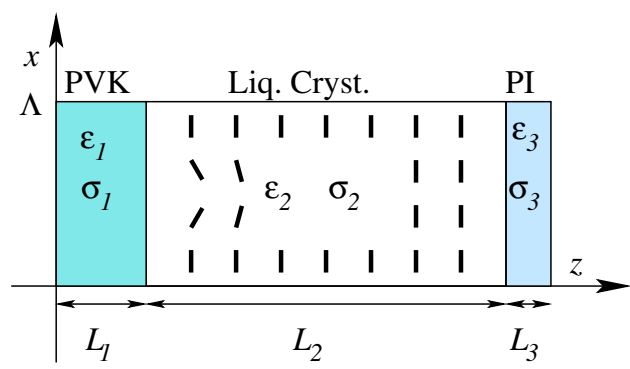

Fig. 8. Schematic diagram of a photorefractive LC cell for the purpose of modeling its electrical behavior. Each layer is assumed to be an isotropic dielectric with nondimensional dielectric permittivity $\epsilon_{k}$, $k=1,2,3$, and conductivity $\sigma_{k}$. The conductance of the PVK: $\mathrm{C}_{60}$ layer has a small spatial modulation. See Appendix A for the full definition and scaling of the parameters. 
PVK: $\mathrm{C}_{60}$ conductivity [Eqs. (A6) and (A9)]. This, finally, gives us the estimate (A10) of the $\overline{\mathrm{MTF}}$, which we plot in Fig. 9 for a fixed voltage applied to the device. The parameters used in this plot were based partly on the estimates derived from impedance measurements reported in [25], and partly on values found in the literature for the conductivity of E7 [33,34] and PI [35].

The graph of the MTF in Fig. 9 is "universal": it is roughly independent of the type of LC used in the cell (the main effect of changing the LC is to change the frequency scale), and different photoconducting layers correspond to different points in the plot. For example, TL205 has a very small conductivity [16]. We have not been able to find precise estimates in the literature, but a value of $\tilde{\sigma}_{2} \simeq 10^{-10} \mathrm{~S} / \mathrm{m}$ is compatible with our results: in this case $\omega=1$ corresponds to a frequency of approximately $2 \mathrm{~Hz}$. In the case of E7, instead, the conductivity is approximately $\tilde{\sigma}_{2} \simeq 10^{-8} \mathrm{~S} / \mathrm{m}$ and a value of $\omega=1$ corresponds to a frequency of approximately $200 \mathrm{~Hz}$.

The first observation to be made is that the MTF is smaller than would be expected from a resistance ladder method that neglects lateral currents: even for the larger pitch considered here, $\Lambda=18 \mu \mathrm{m}$, its maximum is approximately $10 \%$, while in a resistance ladder model for the parameters used in Fig. 9 the maximum modulation is approximately $25 \%$. The difference between the two values is the effects of the lateral currents, mainly in the thicker LC layer, that limit the effect of the conductivity modulation. If we relax the hypothesis that the modulation of the conductivity of the polymer layer is small, numerical simulations of Eq. (A1) show that it is possible to obtain larger voltage modulations across the LC. However, the limiting effect of the lateral currents on the pitch remains.

The second observation is that the amplitude of the modulation is significant only for relative conductivities of the LC-PVK: $\mathrm{C}_{60}$ layers that are within two orders of magnitude of each other, i.e., when the resistance of the PVK: $\mathrm{C}_{60}$ and of the LC layers is comparable. The modulation disappears at high frequency because the LC layer acts as a large capacitance and the voltage is entirely dropped across it. The lowfrequency decrease visible in Fig. 9 is due to the fact that part of the DC voltage is dropped across the PI layer.

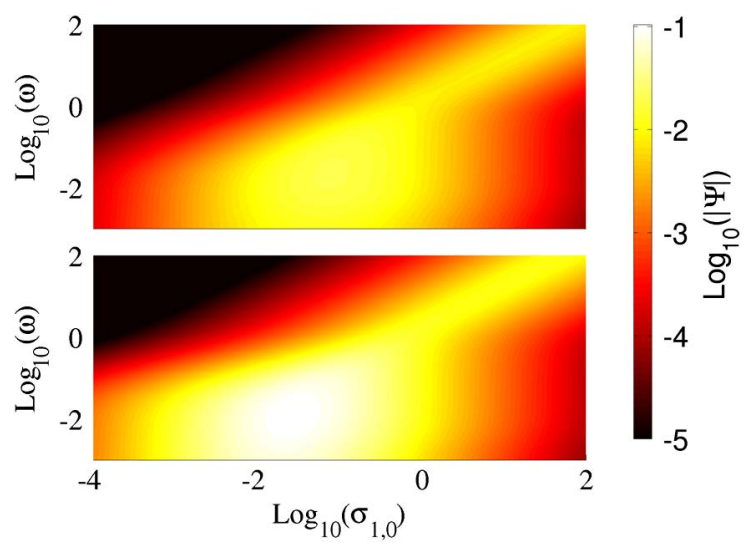

Fig. 9. Modulation transfer function as a function of nondimensional frequency and conductivity of the PVK: $\mathrm{C}_{60}$. The top and bottom plots correspond to grating spacing $\Lambda=\{2,18\} \mu \mathrm{m}$, respectively. Cell parameters: $L_{1}=150 \mathrm{~nm}, L_{2}=12 \mu \mathrm{m}, L_{3}=10 \mathrm{~nm}, \epsilon_{1}=\epsilon_{3}=3 \epsilon_{0}$, $\epsilon_{2}=6 \epsilon_{0}, \sigma_{3}=10^{-3}$, and $\sigma_{1,1}=0.1$. Assuming a LC conductivity of approximately $\tilde{\sigma}_{2} \simeq 10^{-10} \mathrm{~S} / \mathrm{m}$, a reasonable estimate for TL205, a value of $\omega=1$ corresponds to a frequency of approximately $2 \mathrm{~Hz}$.

\section{B. Bragg versus Raman-Nath}

We are now in a position to analyze how the cell properties affect beam coupling. One of the key parameters in many beam-coupling experiments is the grating pitch, $\Lambda$ : narrow pitches (Bragg regime) give larger angles between the beams and reduce their number. Larger pitches (Raman-Nath regime) produce a fan of many beams with small angular separation between them. PVK: $\mathrm{C}_{60}$ is an excellent insulator in the dark: a conductivity value of $1.6 \cdot 10^{-13} \mathrm{~S} / \mathrm{m}$ is reported in [36]. We could, therefore, expect that it should be able to generate a small pitch voltage modulation in the LC. However, the experiments indicate that this is not the case (see Section 2.C).

By looking at Fig. 9 we realize that the electric field modulation at the PVK: $\mathrm{C}_{60}-\mathrm{LC}$ interface is more or less the same in the Bragg (top plot) and Raman-Nath (bottom plot) regimes. The MTF is smaller in the Bragg than in the Raman-Nath regime: as the pitch becomes smaller lateral currents in the LC become more and more effective at balancing the potential difference between neighboring regions. However, the modulation of the electric field, which is ultimately responsible for the refractive index grating, is proportional to $\Delta V_{\mathrm{LC}} / \Lambda$, and this ratio is approximately constant.

In order to understand the effect that the modulation of the applied voltage has on the LC, we need to consider the (nondimensional) LC free energy for the $\mathcal{Q}$-tensor field [37],

$$
\begin{aligned}
\mathcal{F}= & \frac{\xi_{0}^{2}}{2}|\nabla \mathcal{Q}|^{2}+\frac{T_{0}}{2} \operatorname{Tr}\left(\mathcal{Q}^{2}\right)-\sqrt{6} \operatorname{Tr}\left(\mathcal{Q}^{3}\right) \\
& +\frac{1}{2} \operatorname{Tr}^{2}\left(\mathcal{Q}^{2}\right)-\chi_{a} \operatorname{Tr}(\mathcal{Q} \mathcal{E})
\end{aligned}
$$

where the applied electric field $\boldsymbol{E}$ is represented by the traceless symmetric tensor

$$
\mathcal{E}=\sqrt{\frac{3}{2}}\left(\boldsymbol{E} \otimes \boldsymbol{E}-\frac{1}{3}|\boldsymbol{E}|^{2} \boldsymbol{I}\right) .
$$

The parameters in the free energy are the nondimensional elastic constant $\xi_{0}^{2}$, the electrostatic coefficient $\chi_{a}$, and the scaled temperature $T_{0}$. All lengths are scaled to the thickness of the LC layer.

As the pitch is decreased the elastic energy increases as $\Lambda^{-2}$ while the electrostatic energy remains more or less constant. Based on this simple model we can therefore expect that the strength of the modulation of the LC will decrease as $\Lambda^{2}$ and will be much weaker in the Bragg than in the Raman-Nath regime. This result is also supported by the analysis in [26], where the authors derive an analytic expression for the diffraction efficiency as a function of the grating pitch in the limit of small LC reorientation.

The analysis of the pitch dependence of the beam-coupling strength highlights two key factors that determine the strength of beam coupling. The first is the elastic constant of the LC: as the modulation pitch decreases the associated elastic energy increases quadratically and will pose the ultimate limitation on the minimum pitch size. The second key factor that determines the strength of beam coupling is the conductivity of the LC: in conductive LCs lateral currents are more effective at reducing the imposed electrical modulation. This is also exemplified by the photorefractive cells analyzed in [38]. These cells use a photorefractive crystal to generate a modulated 
electric field: the strength of the modulation is stronger in low ionic content LCs, presumably because lateral currents are less able to counteract the electric field modulation. In our cells, instead, a compromise must be reached: in the limit of an insulating LC the modulation of the conductance of the PVK: $\mathrm{C}_{60}$ layer would have no effect, because all the voltage would be, in any case, dropped across the LC (see Fig. 9).

\section{Driving Frequency and Beam-Coupling Strength}

The experimental results illustrated in Fig. 5 indicate that in order to accurately determine the diffraction efficiency of the cell we need to include the voltage dependence of the conductivity of the PVK: $\mathrm{C}_{60}$. It is very hard to measure this in our setup. In the same spirit as Section 3.A we have tested various types of voltage dependence, with more or less rapid transitions between the two conductivity regimes, and neglected the polarity dependence of the PVK: $\mathrm{C}_{60}$ conductivity. Figure 10 shows a typical example. The top panel shows the dependence of the unmodulated PVK: $\mathrm{C}_{60}, \sigma_{1,0}(V)$, for which we have assumed the following: it is a typical hyperbolic tangent transition between two conductivity levels. The bottom panel is a plot of $\Delta V_{L C}$, i.e., the amplitude of the voltage modulation at the $\mathrm{PVK}: \mathrm{C}_{60}-\mathrm{LC}$ interface, as a function of frequency $\omega$ and voltage $V$ applied to the cell.

Figure 10 is not a quantitative interpretation of the beamcoupling data reported in Section 2.B, but holds the key to understanding why there can be two coupling regions. If at low voltage the conductivity of the $\mathrm{PVK}: \mathrm{C}_{60}$ layer is much smaller than that of the LC, we can expect that coupling will occur only for nondimensional frequencies $\omega \lesssim 0.1$ (equivalent to approximately $0.2 \mathrm{~Hz}$ for TL205). At higher voltage the conductivity of the PVK: $\mathrm{C}_{60}$ increases and the beam-coupling regime shifts to higher frequency. It is likely that this scenario applies to TL205, and this could explain why we observe two coupling regions, one at higher and one at lower frequency, for this LC. In the case of E7, a much more conductive LC, it is likely that the conductivity of PVK: $\mathrm{C}_{60}$ remains small for all voltages (i.e., the system is always in the bottom left corner of the bottom panel of Fig. 9) and beam coupling is observed only at low frequencies.
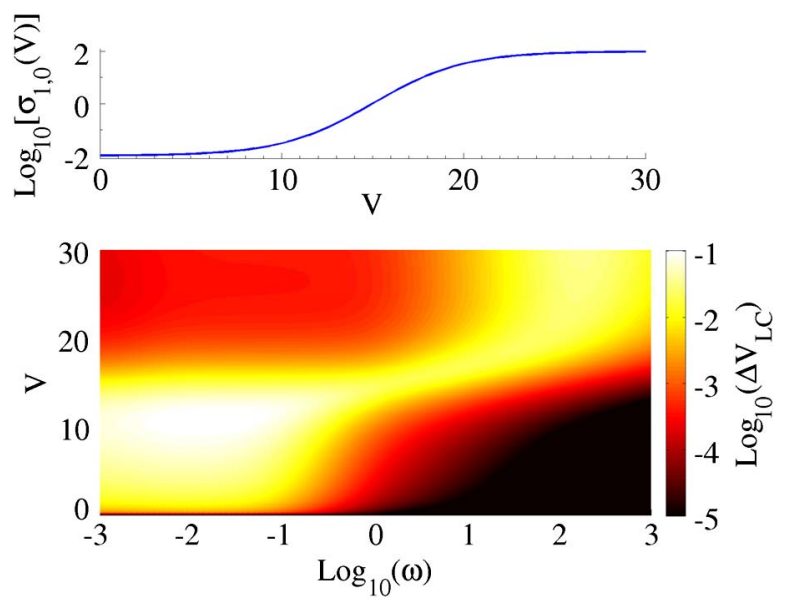

Fig. 10. Voltage conductivity for PVK: $\mathrm{C}_{60}$ (top) used to compute the amplitude of the voltage modulation at the PVK: $\mathrm{C}_{60}-\mathrm{LC}$ interface as a function of frequency and voltage (bottom). Cell parameters as in the bottom panel of Fig. 9 .

\section{CONCLUSIONS}

We have analyzed experimentally and theoretically the extent of beam coupling in photorefractive liquid crystal (LC) cells. The VTF was used to determine experimentally the voltage drop across the LC layer as a function of frequency and light illumination. We have also determined experimentally the optimal range of the beam-coupling existence and magnitude by scanning the voltage-frequency parameter space. The experiment has been performed for cells containing two LCs, E7 and TL205, and for two different grating pitch values. There is no coupling observed for a small grating pitch $(\Lambda \sim 2 \mu \mathrm{m}$ in our system). For a larger grating, i.e., $\Lambda \sim 18 \mu \mathrm{m}$, we identify two regions of the beam-coupling existence: at low frequency and voltage, and at higher frequency and voltage. The beam-coupling strength depends significantly on the conductivity of the LC and is 50 times larger for E7 than for TL205.

The experimental results have been interpreted by making use of the MTF, a "universal-like" function that can approximately characterize the beam-coupling strength independently of the LC used. We have estimated it using a simple electrical model of the photorefractive cell. The MTF, coupled with a model of the LC orientation and of beam interaction, offers a key to interpret the experimental results, namely (i) small pitch gratings entail a large elastic energy cost and are washed out by the director field, (ii) the voltage dependent conductivity of the PVK: $\mathrm{C}_{60}$ gives rise to the two beam-coupling regions in the voltage-frequency parameter space, and (iii) there is an optimal polymer/LC electric conductivity ratio for largest coupling.

Even in the case of a higher conductivity LC such as E7, the MTF is of the order of $10 \%-20 \%$. This, together with the large effective nonlinearity of the photorefractive cell, still produces significant beam coupling, $5 \%$ in our nonoptimized cells. However, it is natural to ask what can be done to increase the MTF upper limit. A larger value of the MTF can be obtained by carefully tuning the photoconducting polymer layer: in the ideal case it should have a large conductivity modulation that makes it switch from being much more to much less resistive than the LC layer as a function of the light illumination. However, such a system will always suffer from limitations of the minimum pitch size. Other options to obtain strong beam coupling are to either modulate the field directly at the photoconductor LC interface (as is done in cells with photorefractive crystals) or change the modulation control of the LC, by, for example, modulating the anchoring (one of the effects discussed in [27]). The first option still suffers from some of the limitations of the photorefractive cells studied here: for example, lateral currents in the LC will partially counteract the effect of the modulated electric field. The second approach is more promising, but photo-aligned layers are normally not reversible. Two recent papers $[39,40]$ study nematic LCs with H-bonded dye-polymer complex, which seem to be promising materials to significantly alter the local properties of LCs using light tuning. Materials like these may lead to direct light modulation of the cell refractive index and allow full exploitation of the enormous beam-coupling potential of LC cells.

\section{APPENDIX A: ELECTRICAL MODEL}

The experimental results reported in Section 2.D and in the literature [41] show that $\mathrm{PVK}: \mathrm{C}_{60}$ is a non-Ohmic conductor. 
In fact, $\mathrm{PVK}: \mathrm{C}_{60}$ has two conductivity states that may differ by six orders of magnitude: it is possible to switch from the low to the high conductivity state by applying a sufficiently large negatively biased field. The switch back is obtained by reversing the bias, with a large hysteresis region [23]. As we do not have a precise electrical characterization of the $\mathrm{PVK}: \mathrm{C}_{60}$ layers used in our cells, we have decided to model it as an Ohmic resistor, with the understanding that the parameter values of the model depend on the voltage: in particular it will be necessary to use different parameter values in the low- and high-voltage regimes. A similar approach was used in [42], where a simple resistance ladder model was used to guide the optimization of a polymer-based LC light valve.

Simple impedance ladder models of the cell are intuitive and provide insight into the system [14]. However, they are not sufficient to calculate the voltage drop across the LC accurately because they neglect the components of the electric current in the direction of the grating: these tend to reduce the potential difference between neighboring regions and, hence, reduce the strength of the voltage modulation. To improve on this, we solve Maxwell's equations to find the electric field at low frequency throughout the cell. By taking the divergence of Ampère's law we obtain

$$
\nabla \cdot[(\tilde{\sigma}+i \tilde{\omega} \tilde{\epsilon}) \nabla \tilde{\psi}]=0
$$

where the superscript indicates a dimensional variable (in SI units). In particular, $\tilde{\psi}$ is the electric potential, $\tilde{\sigma}$ is the conductivity of the medium, and $\tilde{\epsilon}$ is the low-frequency dielectric permittivity (assumed for simplicity to be isotropic). We have imposed a time dependence $e^{i \tilde{\omega} \tilde{t}}$ for the electric field, with $\tilde{\omega}$ the AC frequency of the field driving the cell. We have solved Eq. (A1) numerically to analyze the case of large conductivity modulation. However, it is more intuitive to find an approximate expression for the electric field throughout the cell under the assumption that the modulation of the conductance of the PVK: $\mathrm{C}_{60}$ layer is small. We solve Eq. (A1) in the PVK: $\mathrm{C}_{60}$, PI, and LC layers (see Fig. 8) assuming that the periodic modulation of the conductance of the $\mathrm{PVK}: \mathrm{C}_{60}$ layer is small. Before proceeding further we nondimensionalize all the variables in Eq. (A1). We indicate nondimensional quantities with the same symbol as their dimensional counterparts, but without the superscript tilde. The voltage $\psi$ is scaled to a reference voltage $\tilde{\psi}_{0}$. The dielectric permittivities of the three layers $\epsilon_{j}$, where $j=1,2,3$ denotes the PVK: $\mathrm{C}_{60}, \mathrm{LC}$, and PI layers, are scaled to the dielectric permittivity of the (assumed isotropic) LC layer $\tilde{\epsilon}_{2}$. The layer thicknesses $L_{j}$ are scaled to the thickness $\tilde{L}$ of the cell. The same scaling applies to the spatial coordinates $(x, z)$, with $z$ and $x$ being into and parallel to the cell, respectively. We indicate the (nondimensional) conductance of the PVK: $\mathrm{C}_{60}$ layer with

$$
\sigma_{1}=\sigma_{1,0}\left[\psi_{1}\left(L_{1}\right)\right]\left[1+\eta \sigma_{1,1} \cos \left(K_{g} x\right)\right]
$$

where $\psi_{1}\left(L_{1}\right)$ is the (nondimensional) voltage dropped across the PVK: $\mathrm{C}_{60}$ layer, $K_{g}=2 \pi / \Lambda, \Lambda$ is the grating period scaled to $\tilde{L}$, and $\eta \ll 1$ is a smallness parameter introduced for book-keeping purposes and that can ultimately be set to 1 . The conductivities $\sigma_{2}$ and $\sigma_{3}$ of the other layers are assumed to be constant. All of them are scaled to the (dimensional) conductivity of the LC layer, $\tilde{\sigma}_{2}$. The scaling of the conductivity and permittivity gives us the nondimensional frequency $\omega=\tilde{\epsilon}_{2} \tilde{\omega} / \tilde{\sigma}_{2}$. We assume a power series solution of the form

$$
\psi_{j}=\psi_{j, 0}(z)+\eta \psi_{j, 1}(z) \cos \left(K_{g} x\right)+O\left(\eta^{2}\right)
$$

where $j=1,2,3$ denotes the PVK: $\mathrm{C}_{60}, \mathrm{LC}$, and PI layers, respectively. To leading order in $\eta$ the problem reduces to solving $\psi_{j, 0}^{\prime \prime}=0$, subject to the boundary conditions that the potential is continuous at the layer boundaries

$$
\begin{aligned}
& \psi_{1,0}(0)=V, \quad \psi_{1,0}\left(L_{1}\right)=\psi_{2,0}\left(L_{1}\right), \\
& \psi_{2,0}\left(1-L_{3}\right)=\psi_{3,0}\left(1-L_{3}\right), \quad \psi_{3,0}(1)=0,
\end{aligned}
$$

and that current is conserved,

$$
\begin{aligned}
& \left(\sigma_{1,0}+i \omega \epsilon_{1}\right) \psi_{1,0}^{\prime}\left(L_{1}\right)=(1+i \omega) \psi_{2,0}^{\prime}\left(L_{1}\right), \\
& (1+i \omega) \psi_{2,0}^{\prime}\left(1-L_{3}\right)=\left(\sigma_{3}+i \omega \epsilon_{3}\right) \psi_{3,0}^{\prime}\left(1-L_{3}\right) .
\end{aligned}
$$

This leading order problem has a solution

$$
\begin{array}{ll}
\psi_{1,0}=V \frac{Z_{1}\left(L_{1}-z\right) / L_{1}+Z_{2}+Z_{3}}{Z_{T}}, \quad 0 \leq z \leq L_{1}, \\
\psi_{2,0}=V \frac{Z_{2}\left(L_{1}-z\right) / L_{2}+Z_{2}+Z_{3}}{Z_{T}}, \quad L_{1} \leq z \leq 1-L_{3}, \\
\psi_{3,0}=V \frac{1-z}{L_{3}} \frac{Z_{3}}{Z_{T}}, \quad 1-L_{3} \leq z \leq 1,
\end{array}
$$

where $Z_{j}=L_{j}\left(\sigma_{j}+i \omega \epsilon_{j}\right)^{-1}$ and the total impedance is given by $Z_{T}=Z_{1}+Z_{2}+Z_{3}$. Expanding to first order in $\eta$ we obtain

$$
\frac{d^{2}}{d z^{2}} \psi_{j, 1}-K_{g}^{2} \psi_{j, 1}=0, \quad j=1,2,3
$$

with boundary conditions

$$
\begin{aligned}
& \psi_{1,1}(0)=\psi_{3,1}(1)=0, \quad \psi_{1,1}\left(L_{1}\right)=\psi_{2,1}\left(L_{1}\right) \\
& \psi_{2,1}\left(1-L_{3}\right)=\psi_{3,1}\left(1-L_{3}\right) \\
& \left(\sigma_{1,0}+i \epsilon_{1} \omega\right) \psi_{1,1}^{\prime}\left(L_{1}\right)+\sigma_{1,0} \sigma_{1,1} \psi_{1,0}^{\prime}\left(L_{1}\right)=(1+i \omega) \psi_{2,1}^{\prime}\left(L_{1}\right), \\
& (1+i \omega) \psi_{2,1}^{\prime}\left(1-L_{3}\right)=\left(\sigma_{3}+i \epsilon_{3} \omega\right) \psi_{3,1}^{\prime}\left(1-L_{3}\right)
\end{aligned}
$$

The order one solution is

$$
\begin{aligned}
\psi_{1,1}= & A_{1} e^{-K_{g} L_{1}} \sinh \left(K_{g} z\right), \quad 0 \leq z \leq L_{1}, \\
\psi_{2,1}= & A_{2} e^{-K_{g} L_{2}} \sinh \left[K_{g}\left(1-L_{3}-z\right)\right] \\
& +B_{2} e^{-K_{g} L_{2}} \sinh \left[K_{g}\left(z-L_{1}\right)\right], \quad L_{1} \leq z \leq 1-L_{3}, \\
\psi_{3,1}= & A_{3} e^{-K_{g} L_{3}} \sinh \left[K_{g}(1-z)\right], \quad 1-L_{3} \leq z \leq 1,
\end{aligned}
$$

where the unknown constants $A_{1}, A_{2}, A_{3}$, and $B_{2}$ are found by substituting Eqs. (A9) into the conditions (A8) and solving the resulting linear system. The final expression is rather long, but not hard to derive. As it is not very informative, it is not reproduced here. In terms of the parameter of this model the MTF is

$$
\Psi=\frac{A_{2}}{\sigma_{1,1}} e^{-K_{g} L_{2}} \sinh \left(K_{g} L_{2}\right),
$$


where we have set the book-keeping parameter $\eta=1$. Note that $A_{2}$ may depend on the voltage applied to the device if we need to take into account the non-Ohmic behavior of the PVK: $\mathrm{C}_{60}$ layer, as is the case in Fig. 10.

\section{REFERENCES}

1. K. Komorowska, A. Miniewicz, and J. Parka, "Holographic grating recording in large area photoconducting liquid crystal panels," Synth. Met. 109, 189-193 (2000).

2. U. Bortolozzo, S. Residori, and J. P. Huignard, "Beam coupling in photorefractive liquid crystal light valves," J. Phys: Appl Phys 41, 224007 (2008).

3. G. Cook, C. A. Wyres, M. J. Deer, and D. C. Jones, "Hybrid organicinorganic photorefractives," Proc. SPIE 5213, 63-77 (2003).

4. A. Miniewicz, "Photorefractive-like all-optical switching in nematic-photoconducting polymer liquid crystal cell," Mol. Cryst. Liq. Cryst. 489, 119-134 (2008).

5. J. Mun, C. S. Yoon, H.-W. Kim, S.-A. Choi, and J.-D. Kim, "Transport and trapping of photocharges in liquid crystals placed between photoconductive polymer layers," Appl. Phys. Lett. 79, 1933-1935 (2001).

6. L. Solymar, D. J. Webb, and A. Grunnet-Jepsen, The Physics and Applications of Photorefractive Materials (Oxford, 1996).

7. M. Kaczmarek and R. Eason, "Near-infrared incoherent coupling and photorefractive response time of 'blue' $\mathrm{Rh}: \mathrm{BaTiO}_{3}$," Opt. Commun. 136, 277-282 (1997).

8. F. Yao, Y. Pei, Y. Zhang, J. Zhang, C. Hou, and X. Sun, "Highresolution photorefractive gratings in nematic liquid crystals sandwiched with photoconductive polymer film," Appl. Phys. B 92, 573-576 (2008).

9. A. Redler and H.-S. Kitzerow, "Influence of doping on the photorefractive properties of a polymer-dispersed liquid crystal," Appl. Phys. Lett. 93, 183304 (2008).

10. M. Talarico and A. Golemme, "Photorefractive binary system based on ferroelectric liquid crystals," J. Phys. Chem. C 111, 7834-7840 (2007).

11. T. Fears, C. Anderson, and J. Winiarz, "Photorefractivity in a polymeric composite photosensitized with NiS nanocrystals," J. Chem. Phys. 129, 154704 (2008).

12. D. Jiang, L. Ding, J. Huang, E. Gu, L. Liu, Z. Chai, and D. Liu, "Synthesis and characterization of photorefractive polymer based on chemically hybridized CdS-PVK nanocomposite with a new azo chromophore," Polymer 48, 7156-7162 (2007).

13. M. Kaczmarek, A. Dyadyusha, S. Slussarenko, and I. C. Khoo, "The role of surface charge field in two-beam coupling in liquid crystal cells with photoconducting polymer layers," J. Appl. Phys. 96, 2616-2623 (2004)

14. M. Herrington, K. Daly, O. Buchnev, G. D'Alessandro, and M. Kaczmarek, "AC-field-enhanced beam coupling in photorefractive, hybrid liquid crystals," Europhys. Lett. 95, 14003 (2011).

15. J. Bateman, M. Proctor, O. Buchnev, N. Podoliak, G. D'Alessandro, and M. Kaczmarek, "Voltage transfer function as an optical method to characterize electrical properties of liquid crystal devices," Opt. Lett. 39, 3756-3759 (2014).

16. G. Cook, A. V. Glushchenko, V. Reshetnyak, A. T. Griffith, M. A. Saleh, and D. R. Evans, "Nanoparticle doped organic-inorganic hybridphotorefractives,” Opt. Express 16, 4015-4022 (2008).

17. T. K. Gaylord and M. G. Moharam, "Thin and thick gratings: terminology clarification," Appl. Opt. 20, 3271-3273 (1981).

18. Y. Wang, "Photoconductivity of fullerene-doped polymers," Nature 356, 585-587 (1992)

19. F. Li, Y. Li, Z. Guo, C. Long, F. Bai, and D. Zhu, "Photoconductivity of 1,2-(1',1',2',2'-Tetracyanomethanoxymethano)[60]fullerene-Doped PVK," J. Appl. Polym. Sci. 72, 209-213 (1999).

20. P. D'Angelo, M. Barra, A. Cassinese, M. Maglione, P. Vacca, C. Minarini, and A. Rubino, "Electrical transport properties characterization of PVK (poly N-vinylcarbazole) for electroluminescent devices applications," Solid-State Electron. 51, 123-129 (2007).

21. J. H. Ham, J. H. Jung, H. J. Kim, D. U. Lee, and T. W. Kim, "Electrical properties and operating mechanisms of nonvolatile organic memory devices fabricated utilizing hybrid poly ( $N$-vinylcarbazole) and $\mathrm{C}_{60}$ composites," Jpn. J. Appl. Phys. 47, 4988-4991 (2008).
22. K. R. Daly, N. Podoliak, O. Buchnev, M. Kaczmarek, and G. D'Alessandro, "Optimal liquid crystal modulation controlled by surface alignment and anchoring strength," J. Opt. Soc. Am. B 29, 2166-2175 (2012).

23. Y.-S. Lai, C.-H. Tu, D.-L. Kwong, and J. Chen, "Charge-transport characteristics in bistable resistive Poly(N-vinylcarbazole) films," IEEE Electron Device Lett. 27, 451-453 (2006).

24. S. B. Abbott, K. R. Daly, G. D’Alessandro, M. Kaczmarek, and D. C. Smith, "Photorefractive control of surface plasmon polaritons in a hybrid liquid crystal cell," Opt. Lett. 37, 2436-2438 (2012).

25. S. B. Abbott, K. R. Daly, G. D'Alessandro, M. Kaczmarek, and D. C. Smith, "A hybrid liquid crystal photorefractive system for the photorefractive coupling of surface plasmon polaritons," J. Opt. Soc. Am. B 29, 1947-1958 (2012).

26. V. O. Kubytskyi, V. Y. Reshetnyak, T. J. Sluckin, and S. J. Cox, "Theory of surface-potential-mediated photorefractivelike effects in liquid crystals," Phys. Rev. E 79, 011703 (2009).

27. V. Y. Reshetnyak, I. P. Pinkevych, G. Cook, D. R. Evans, and T. J. Sluckin, "Two-beam energy exchange in a hybrid photorefractive-flexoelectric liquid-crystal cell," Phys. Rev. E 81, 031705 (2010).

28. T. K. Däubler, L. Kulikovsky, D. Neher, V. Cimrová, J. C. Hummelen, E. Mecher, R. Bittner, and K. Meerholz, "Photoconductivity and charge-carrier photogeneration in photorefractive polymers," Proc. SPIE 4462, 206-216 (2002).

29. M. Scalerandi, P. Pagliusi, G. Cipparrone, and G. Barbero, "Influence of the ions on the dynamical response of a nematic cell submitted to a dc voltage," Phys. Rev. E 69, 051708 (2004).

30. F. C. M. Freire, A. L. Alexe-Ionescu, M. Scalerandi, and G. Barbero, "Voltage decay time of a liquid crystal cell submitted to a large difference of potential," Appl. Phys. Lett. 89, 214101 (2006)

31. A. L. Alexe-Ionescu, G. Barbero, F. Ciuchi, A. Mazzulla, and N. Scaramuzza, "Surface treatment and bulk density of ions in nematic liquid crystals," J. Appl. Phys. 106, 044508 (2009).

32. G. Barbero, G. Cipparrone, O. G. Martins, P. Pagliusi, and A. M. Figueiredo Neto, "Electrical response of a liquid crystal cell: the role of Debye's layer," Appl. Phys. Lett. 89, 132901 (2006).

33. I. Dierking, G. Scalia, and P. Morales, "Liquid crystal-carbon nanotube dispersions," J. Appl. Phys. 97, 044309 (2005).

34. S. Tomylko, O. Yaroshchuk, O. Kovalchuk, U. Maschke, and R. Yamaguchi, "Dielectric properties of nematic liquid crystal modified with diamond nanoparticles," Ukr. J. Phys. 57, 239-243 (2012)

35. S. Chisca, I. Sava, V. Musteata, and M. Bruma, "Dielectric and conduction properties of polyimide films," in International Semiconductor Conference (CAS) (2011), Vol. 2, pp. 253-256.

36. S. Santos, C. Gómez, and I. Salazar, "DC Conductivity in polyn-vinylcarbazole: a study of dielectric measurements," Int. J. Polym. Mater. 51, 567-575 (2002).

37. K. R. Daly, G. D'Alessandro, and M. Kaczmarek, "An efficient $\mathcal{Q}$-tensor-based algorithm for liquid crystal alignment away from defects," SIAM J. Appl. Math. 70, 2844-2860 (2010).

38. D. E. R. L. Sutherland and G. Cook, "Determination of large nematic pre-tilt in liquid crystal cells with mechanically rubbed photorefractive Ce:SBN windows," Opt. Express 14, 5365-5375 (2006).

39. E. Ouskova, J. Vapaavuori, and M. Kaivola, "Self-orienting liquid crystal doped with polymer-azo-dye complex," Opt. Mater. Express 1, 1463-1470 (2011).

40. A. Uklein, A. Vasko, E. Ouskova, M. Brodyn, and V. Gayvoronsky, "Nonlinear optical properties of new photosensitive smart materials based on nematic liquid crystal with h-bonded dye-polymer complex," Opt. Commun. 296, 79-83 (2013).

41. E. Hendrickx, Y. Zhang, K. B. Ferrio, J. A. Herlocker, J. Anderson, N. R. Armstrong, E. A. Mash, A. P. Persoons, N. Peyghambarian, and B. Kippelen, "Photoconductive properties of PVK-based photorefractive polymer composites doped with fluorinated styrene chromophores," J. Mater. Chem. 9, 2251-2258 (1999).

42. N. Collings, T. D. Wilkinson, A. Jeziorska, A. Davey, B. Movaghar, and W. A. Crossland, "Charge-injecting layers for liquid crystal light valves," in Photonics Europe (International Society for Optics and Photonics, 2004), pp. 421-427. 